\title{
Long-Range Propagation and Interference of $d$-Wave Superconducting Pairs in Graphene
}

\author{
D. Perconte, ${ }^{1,2}$ K. Seurre $\odot,{ }^{1}$ V. Humbert, ${ }^{1}$ C. Ulysse, ${ }^{3}$ A. Sander, ${ }^{1}$ J. Trastoy, ${ }^{1}$ V. Zatko $\odot,{ }^{1}$ F. Godel $\odot,{ }^{1}$ P. R. Kidambi, ${ }^{4,5}$ \\ S. Hofmann, ${ }^{5}$ X. P. Zhang, ${ }^{6,7}$ D. Bercioux $\odot,{ }^{7,8}$ F. S. Bergeret $\odot,{ }^{6,7}$ B. Dlubak $\odot,{ }^{1}$ P. Seneor, ${ }^{1}$ and Javier E. Villegas ${ }^{1, *}$ \\ ${ }^{1}$ Unité Mixte de Physique, CNRS, Thales, Université Paris-Saclay, 91767 Palaiseau, France \\ ${ }^{2}$ Laboratorio de Bajas Temperaturas y Altos Campos Magnéticos, Departamento de Física de la Materia Condensada, Instituto Nicolás \\ Cabrera and Condensed Matter Physics Center (IFIMAC), Universidad Autónoma de Madrid, E-28049 Madrid, Spain \\ ${ }^{3}$ Centre for Nanoscience and Nanotechnology, CNRS, Université Paris-Sud/Université Paris-Saclay, \\ Boulevard Thomas Gobert, 91120 Palaiseau, France \\ ${ }^{4}$ Department of Chemical and Biomolecular Engineering, Vanderbilt University, \\ 2400 Highland Avenue, Nashville, Tennessee 37212, USA \\ ${ }^{5}$ Department of Engineering, University of Cambridge, Cambridge CB3 OFA, United Kingdom \\ ${ }^{6}$ Centro de Fisica de Materiales (CFM-MPC), Centro Mixto CSIC-UPV/EHU, 20018 Donostia-San Sebastián, Basque Country, Spain \\ ${ }^{7}$ Donostia International Physics Center (DIPC), Manuel de Lardizabal, 4, 20018 Donostia-San Sebastián, Spain \\ ${ }^{8}$ IKERBASQUE, Basque Foundation of Science, 48011 Bilbao, Basque Country, Spain
}

(Received 24 February 2020; revised 22 June 2020; accepted 22 July 2020; published 18 August 2020)

\begin{abstract}
Recent experiments have shown that proximity with high-temperature superconductors induces unconventional superconducting correlations in graphene. Here, we demonstrate that those correlations propagate hundreds of nanometers, allowing for the unique observation of $d$-wave Andreev-pair interferences in $\mathrm{YBa}_{2} \mathrm{Cu}_{3} \mathrm{O}_{7}$-graphene devices that behave as a Fabry-Perot cavity. The interferences show as a series of pronounced conductance oscillations analogous to those originally predicted by de Gennes-Saint-James for conventional metal-superconductor junctions. The present demonstration is pivotal to the study of exotic directional effects expected for nodal superconductivity in Dirac materials.
\end{abstract}

DOI: 10.1103/PhysRevLett.125.087002

The superconducting proximity effect in graphene has attracted much interest since the pioneering experiments [1]. This roots down to the graphene's electronic structure, which strongly affects the underlying mechanisms: Andreev reflection and coherent propagation of electronhole pairs [2]. A distinctive feature is the strong dependence of the proximity behavior on the graphene's doping level, which dramatically changes the Andreev reflection $[3,4]$ as compared to metals. Other unique features include gate [5] or magnetic-field [6,7] driven transitions from bulk to edge transport. Studies on graphene have also paved the way for understanding the proximity effect in other Dirac materials, such as topological insulators [8-11].

Experiments have mostly focused on conventional low critical temperature $\left(T_{C}\right)$ superconductors with $s$-wave pairing. Despite early theoretical studies showing that $d$-wave (high- $T_{C}$ ) superconductors should lead to novel directional effects [12,13] and exotic pairing [14,15], evidence for unconventional superconductivity in graphene has been found only recently $[16,17]$. Scanning electron tunneling microscopy (STM) of graphene on $\mathrm{Pr}_{2-x} \mathrm{Ce}_{x} \mathrm{CuO}_{4}$ revealed a superconducting gap [16] and spectral features suggesting ( $p$-wave) superconductivity induced in graphene. Experiments on $\mathrm{YBa}_{2} \mathrm{Cu}_{3} \mathrm{O}_{7}$ (YBCO) and chemical-vapordeposited (CVD) graphene devices by some of us [17] showed transparent superconductor-graphene interfaces and clear evidence of Andreev reflection. Interestingly, we also found that the Andreev electron-hole pair transmission can be modulated by a back-gate voltage, through a mechanism analogous to the Klein tunneling [18]. However, neither the STM experiments nor ours on solid-state devices probed the length scale over which the unconventional correlations penetrate into graphene.

Here, we demonstrate the long-range propagation of unconventional superconductivity into graphene via the observation of $d$-wave Andreev-pair interferences. These manifest themselves as conductance oscillations in devices that allow confining Andreev pairs within a graphene "cavity" whose length $L$ is up to a few hundred nanometers. Predicted for proximitized graphene homojunctions $[12,13]$, the oscillations are analogous to the de Gennes-Saint-James [19] and McMillan-Rowel resonances [20] in the electronic density of states of ultrathin normal metals backed by superconductors. Figure 1(a) displays a cartoon of the underlying mechanism. Electrons injected in the cavity are Andreev reflected as holes at the interface with the superconductor, retrace their path to the opposite cavity's end where they are normal reflected, and travel back toward the superconductor, where they are again Andreev reflected (now as electrons). This results in destructive-constructive interferences dictated by the energy-dependent phase accumulated along the loop. This phenomenon shows up as conductance oscillations as a function of the bias voltage $V_{\text {BIAS. }}$. Because Andreev pairs stemming from YBCO have 


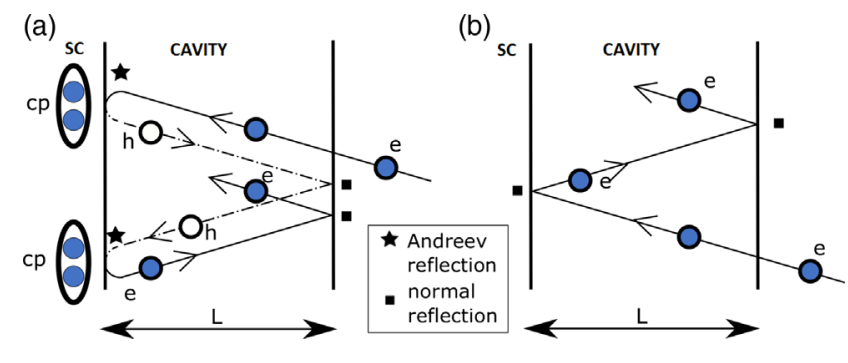

FIG. 1. Interferences in a proximitized cavity (a) An electron Andreev reflected at the SC-cavity interface propagates as a hole back to the other end, where it is normal reflected toward the SC-cavity interface to undergo again Andreev reflection (AR). The lower AR is the time reversal process of the upper one. (b) The electron can also be normal reflected at the SC cavity, travel back to the other cavity interface to be again normal reflected. This process results in Fabry-Perot resonances.

$d$-wave symmetry, they decay over distances comparable to the mean free path $l[21,22]$. Thus, the observation of Andreev-pair interferences implies ballistic or quasiballistic transport. Consistently, the Andreev-pair interferences are accompanied by normal-electron resonances that result from normal reflections at the two cavity's ends [sketch in Fig. 1(b)] and commensurability between $L$ and the electrons' wavelength [18,23-26]. These different resonances result in oscillations as a function of both the gate $V_{G}$ and bias $V_{\mathrm{BIAS}}$, and can be distinguished from Andreev pairs related ones by their distinct periodicity and voltage regime in which they dominate. To our knowledge, the concurrent observation of both resonances had been restricted to junctions between $s$-wave superconductors and topological insulators $[9,10]$, in which Andreev-pair interferences manifest at very low temperatures as a function of the gate voltage $V_{G}$. Here, they can be observed at higher temperatures and show in a rich series of oscillations as a function of $V_{\text {BIAS }}$ because of the high- $T_{C}$ and large gap of YBCO (tens of $\mathrm{meV}$ ) which, contrary to low-temperature superconductors [27], may enclose many orders of interference.

The planar devices [Fig. 2(a)] consist of four superconducting (SC) $c$-axis oriented $\mathrm{YBCO}_{50 \mathrm{~nm}} / \mathrm{Au}_{4 \mathrm{~nm}}$ electrodes disposed within an insulating (INS) YBCO matrix and separated by a gap of length $100 \mathrm{~nm}<L<800 \mathrm{~nm}$. Asgrown YBCO films showed $T_{C} \sim 90 \mathrm{~K}$ while $T_{C} \sim 70 \mathrm{~K}$ for lithographed devices, indicating moderate deoxygenation during fabrication. The ultrathin Au interlayer is deposited in situ to preserve the superconducting properties and improve the interfacial transparency [17]. The Au interlayer is crucial: none of the effects reported here could be observed in its absence. Notice that it is one order of magnitude thinner than the mean free path in $\mathrm{Au}$, and consequently $d$-wave correlations expectedly propagate undisturbed across it [21,22]. A single-layer CVD grown [28] graphene bridge $(G)$ connects the four electrodes, each of which terminates in a gold pad [1-4 in Fig. 2(b)] used for wire bonding. The transparency of the electrical contact between each SC
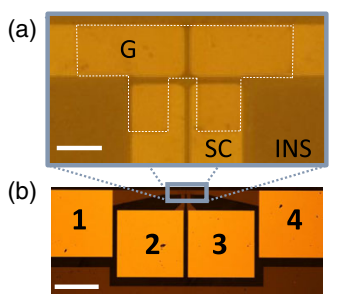

(c)
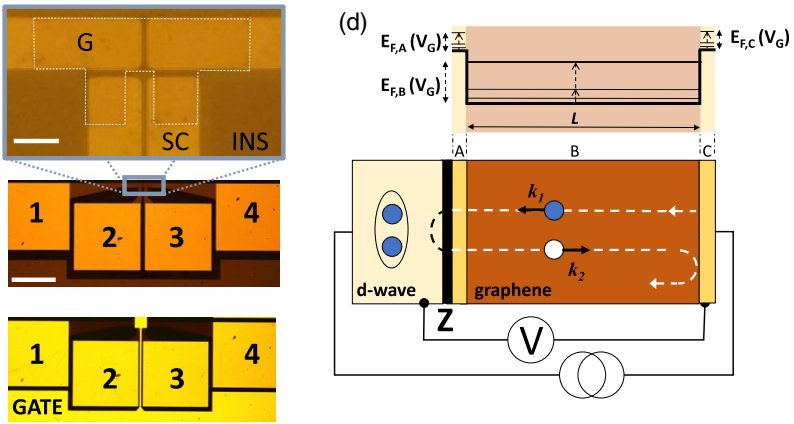

FIG. 2. (a),(b) Micrograph of a graphene-YBCO superconducting device. The scale bar is $10 \mu \mathrm{m}$. (a) shows a zoom on the junction: Superconducting electrodes (SC) are connected by graphene bridges $(G)$ on top of insulating YBCO (INS). The scale bar is $100 \mu \mathrm{m}$. (c) Micrograph of a device with the top gate. (d) Scheme of the graphene homojunction model. The doping depends on whether graphene lies on insulating or superconducting $\mathrm{YBCO}$, which results in three graphene regions $A$, $B, C$ with different Fermi energy. This creates the cavity where interferences occur. The lower scheme shows the equivalent electrical circuit. The YBCO-Au-graphene interface, characterized by a barrier strength $Z$, is measured in series with the cavity.

electrode and the graphene varied largely, even within a single device. Here, we report on effects that arise only at high transparency contacts, which is often the case for just one out of four contacts per device. The structure is covered by a $45 \mathrm{~nm}$ thick $\mathrm{AlO}_{x}$ layer (gate dielectric) [29]. As reported earlier [30], with this encapsulation the carrier mobility in the used CVD graphene reaches up to $\mu \sim 7000 \mathrm{~cm}^{2} \mathrm{~V}^{-1} \mathrm{~s}^{-1}$. The top gate is made of $\mathrm{Au}$ entirely [Fig. 2(c)] and the gate capacitance as measured over insulating $\mathrm{YBCO}$ is $C_{G} \sim 5 \times 10^{11} e \mathrm{~cm}^{-2} \mathrm{~V}^{-1}$ (with $e$ the electron charge). Further details are reported elsewhere [17].

We performed three-probe measurements, with the current $I$ injected e.g., from 1 to contact 4 and the $V_{\text {BIAS }}$ measured between 1 and 3. Thus, we probe the YBCO-Au-graphene interface in series with the graphene "cavity" formed between the SC electrodes, as sketched in Fig. 2(d). The wiring as well as Au-YBCO contact resistances are Ohmic and negligible [17]. The differential conductance $G\left(V_{\mathrm{BIAS}}\right)=$ $d I / d V_{\text {BIAS }}$ is measured using a Keithley 6221 current source coupled to a Keithley 2182.

Examples of low-temperature $(3.2 \mathrm{~K})$ differential conductance measurements are shown in Figs. 3(a), 3(d), and 4(a). $G\left(V_{\text {BIAS }}\right)$ shows a low-bias feature that stands out from the high-bias conductance level. That is either a conductance decrease [Fig. 3(d)] or an enhancement [Fig. 4(a)] within a typical bias range $\left|V_{\text {BIAS }}\right|<\delta_{\text {exp }} \sim 20-70 \mathrm{mV}$ highlighted by the gray shade. Those features are reminiscent of the conductance decrease/increase observed for $e\left|V_{\mathrm{BIAS}}\right|<\Delta$ in superconducting-normal-metal junctions depending on the interface transparency [31]. However, here they extend over a bias range $\delta_{\exp }$ that clearly exceeds the superconducting energy gap expected for moderately 

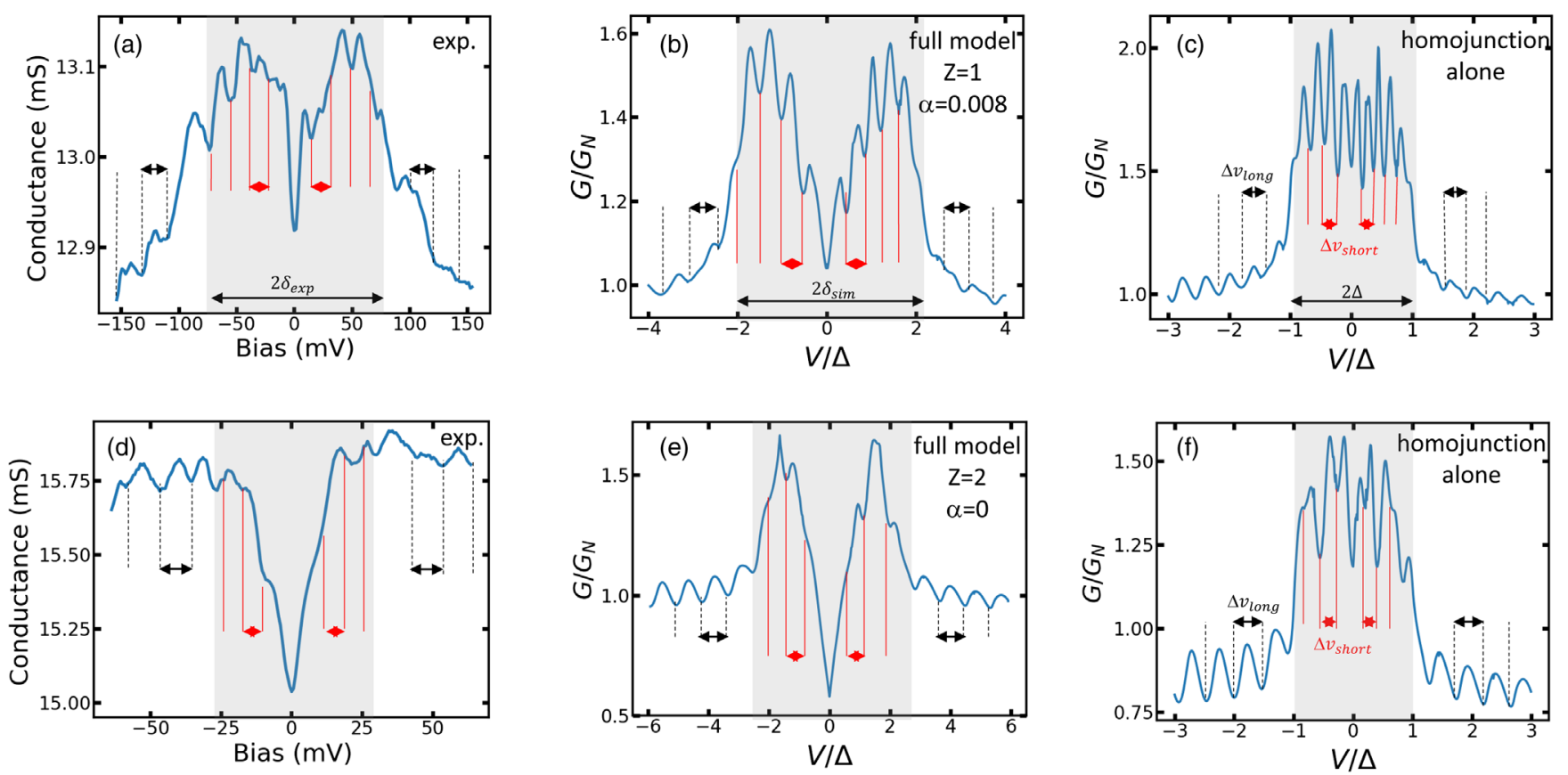

FIG. 3. (a),(d) Conductance versus $V_{\text {bias }}$ for two different devices, $B 3 U$ (a) and $B 4 U$ (b) measured at $3.2 \mathrm{~K}$. Vertical lines point out the series of oscillations, and the horizontal double-headed arrows indicate the periods $V_{\text {long }}$ (black) and $V_{\text {short }}$ (red). The shaded area indicates the width $2 \delta$ of the superconducting-gap related feature. (b) and (e) show corresponding full-model calculations of the conductance with the $Z$ and $\alpha$ indicated in the legend (c) and (f) shows simulations for a proximitized graphene cavity alone, without finite- $Z$ junction in series.

underdoped to optimally doped YBCO, which is reported in the range $\Delta_{\text {YBCO }} \sim 15-30 \mathrm{meV}$ [32-35]. Furthermore, details such as the sharp zero-bias peak observed for some devices (see, e.g., B4D and E3D in the Supplemental Material [36]) are characteristic of junctions involving $d$ wave superconductors, and appear when the $d$-wave nodes form a certain angle with respect to the junction interface $[33,37,38]$.

In addition to the central features, an oscillation pattern appears in Figs. 3(a) and 3(d), that extends over a bias range well above $\delta_{\exp }$. Indeed, two types of oscillations exist: the ones with longer period $V_{\text {long }}$, predominant for $\left|V_{\text {BIAS }}\right|>\delta_{\text {exp }}$, and the other with shorter $V_{\text {short }}$ which are more prominent at $\left|V_{\text {BIAS }}\right|<\delta_{\text {exp }}$.

Figure 4 show an example of gating effects. Figure 4(a) displays $G\left(V_{\text {BIAS }}\right)$ without gate voltage $\left(V_{G}=0\right)$, and shows the general features discussed above. Figure 4(b) is a color plot of a series of $G\left(V_{\mathrm{BIAS}}\right)$ at constant temperature and varying $V_{G}$. The central (red) feature corresponds to the zero-bias conductance peak. The conductance is periodically modulated by $V_{G}$ : a pattern of oblique lines (light-blue-green) indicates that $V_{G}$ gradually "shifts" the oscillations observed as a function of $V_{\text {BIAS }}$. The oblique lines' slope gradually varies with $V_{G}$, which results in pronounced curvature over the plot periphery (low $V_{G}$ or high $V_{\text {BIAS }}$ ).

We discuss now a model that explains the main observations, namely (i) the increase or decrease of the zero-bias conductance; (ii) the origin and period of conductance oscillations. The model is based on the BlonderTinkham-Klapwijk formalism [31] extended to junctions between $d$-wave superconductors and normal metals [39] and to proximitized graphene homojunctions [12,13]. A scheme is shown in Fig. 2(d). First, we consider the
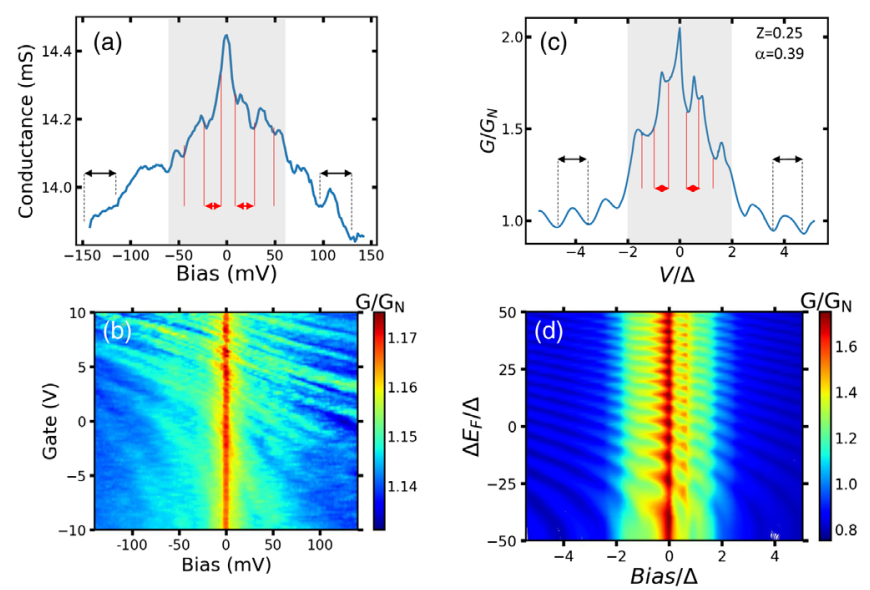

FIG. 4. (a) Conductance versus $V_{\text {bias }}$ for device $A 5 U$, measured at $T=4 \mathrm{~K}$. (b) Same device conductance (color scale) as function of bias voltage (horizontal axis) and gate voltage (vertical axis). (c) Full-model calculation of the conductance with $Z$ and $\alpha$ indicated in the legend (d). Corresponding fullmodel calculation of the conductance as a function of bias and the Fermi energy variation in the cavity. 
YBCO-Au-graphene interface. Because the Au thickness $(\sim 5 \mathrm{~nm})$ is well below the mean free path $l_{\mathrm{Au}} \sim 40 \mathrm{~nm}[40]$ and the low- $T$ coherence length $\xi_{\mathrm{Au}}=\hbar v_{F} l /(6 \pi K T) \sim$ $30 \mathrm{~nm}$ [41], we characterize that interface [black in Fig. 2(d)] via a single Blonder-Tinkham-Klapwijk barrier-strength parameter $Z$. If $Z=0$ the transmission between YBCO and graphene is only mediated by the Andreev reflection. By increasing $Z$, Andreev reflection turns less dominant, normal reflection is enhanced, and transmission is dominated by tunneling. We model the graphene "channel" where Andreev pairs and normal electrons propagate, considering three different regions $A, B, C . A$ and $C$ correspond to graphene lying on superconducting YBCO-Au [yellow in Fig. 2(d)], but we consider that only contact $A$ has a relatively low $Z$. The region $B$ of length $L$ [dark in Fig. 2(d)] lies on insulating YBCO. Here, the Fermi energy $E_{F, B}$ is expectedly different from $E_{F, A}, E_{F, C}$ as the graphene's doping depends on the substrate's electronic properties [42]. Within this model, the structure is a graphene homojunction with a gate-tunable Fermi energy step, as sketched in the upper Fig. 2(d), and behaves as a resonant cavity for electrons and Andreev pairs.

The conductance is calculated numerically by considering the YBCO-Au-graphene interface $[33,39]$ and the proximitized graphene homojunction $[12,13]$ in series (see Supplemental Material for details [36]). The input parameters are $Z$, the reduced Fermi energies $\epsilon_{F, i} \equiv E_{F, i} / \Delta$ (with $i=A, B, C$, and $\Delta$ the energy gap induced in $A$ by proximity effect), the reduced cavity's length $\Lambda \equiv L / \lambda_{F, A}$ (with the Fermi wavelength $\lambda_{F, A}$, chosen as a reference following the original model $[12,13])$, and $\alpha$ the effective angle between $d$-wave antinodes and the homojunction interfaces. The key parameters are $\Lambda$ and $\epsilon_{F, A}$ : they determine $V_{\text {long }}$ and $V_{\text {short }} . \alpha$ and $Z$ determine the conductance background shape, and particularly whether there is a conductance decrease or enhancement around zero bias. We stress that the sharp zero-bias peak observed in some devices cannot be reproduced by the simulation unless we consider $\alpha \neq 0$ (see Supplemental Material [36], Sec. VII). This is a consequence of the $d$-wave symmetry of the correlations propagating across the different interfaces. $\epsilon_{F, B}$ and $\epsilon_{F, C}$ only change the oscillations' phase and amplitude, but do not affect their period [13]. To choose the simulation parameters that yield the best agreement with the experimental curves we proceed as detailed in the Supplemental Material, Secs. 2-4 [36]. Simulation examples are shown in Figs. 3(b), 3(e) and 4(c) for the experiments in Figs. 3(a), 3 (d) and 4(a), respectively. The simulations closely reproduce the main experimental features (see further examples and table of parameters in Supplemental Sections 5-6 [36]).

In order to illustrate the different contributions to the conductance, Figs. 3(c) and 3(f) display the conductance of a proximitized graphene homojunction [13] alone, i.e., without a finite- $Z$ junction in series. The same $\epsilon_{F, i}, \Lambda$, and $\alpha$ as for the full-model calculations are used. Here, the conductance shows a background dependence on $V_{\mathrm{BIAS}}$ very different from the experiments, which evidences that it is strongly influenced by the YBCO-Au-graphene interface. In particular, the zero-bias "dip" only emerges if a finite- $Z$ junction is considered. However, Figs. 3(c) and 3(f) do show the short and long-period oscillations observed experimentally. This demonstrates that they originate within the graphene homojunction. Notice that the ratio between the short and long periods is different from that in the experiments (and full-model simulations): in Figs. 3(c) and 3(f), $V_{\text {short }}$ is clearly shorter (relative to $V_{\text {long }}$ ) than in Figs. 3(a) and 3(b) and in Figs. 3(d) and 3(e). This is because in experiments (and full model) $V_{\text {BIAS }}$ is divided between the graphene homojunction and the YBCO-Augraphene interface, and the strongly nonlinear conductance leads to a voltage distribution that varies depending on the

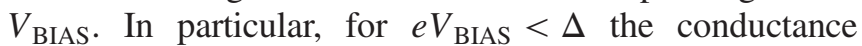
across the YBCO-Au-graphene interface decreases (this is more pronounced for higher $Z$ values) while the homojunction conductance increases [see Figs. 3(c) and 3(f)]. Consequently, $V_{\text {short }}$ (observed for $e V_{\text {BIAS }}<\Delta$ ) appears "stretched" relative to the $V_{\text {long }}$ (observed for $e V_{\text {BIAS }}>\Delta$ ).

The oscillations' physical meaning can be understood from Fig. 5. This displays the short (red circles) and long (black squares) oscillation period as a function of the inverse of the cavity length $L_{\text {device }}^{-1}$ defined upon devices fabrication. Specifically, we plot the period $V=v \Delta$, where $\Delta$ is estimated as discussed in the Supplemental Material [36] and $v$ is the period obtained from our model once the contribution of the YBCO-Au-graphene interface has been removed-as in Figs. 3(c) and 3(e)-to avoid the aforementioned $V_{\text {BIAS }}$ division artifacts.

The long-period oscillations follow $V_{\text {th,long }}=h v_{F} /$ $2 L_{\text {device. }}$. This is as expected from the interference between electrons traveling back and forth from one cavity side to the other after normal reflections [Fig. 1(b)]. This period results [18,23-26] from the interference condition $2 L_{\text {device }} k=2 n \pi$ (with $n$ an integer and $k$ the electron wave

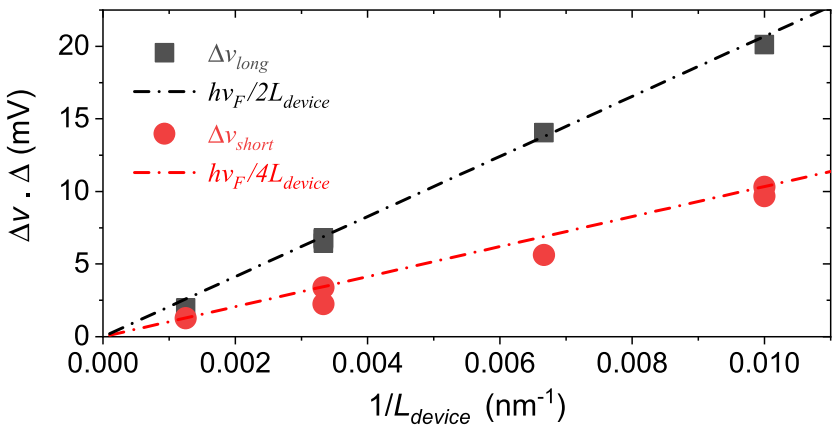

FIG. 5. Oscillations' period as a function of the cavity length $L_{\text {device }}$. The black and red dashed lines respectively correspond to the period theoretically expected for electron (Fabry-Perot) and Andreev-pair (de Gennes-Saint-James) interferences. 
vector) and the graphene's linear dispersion, which yields $V=\hbar v_{F} k$ [43]. These interferences imply normal-electron coherence over $\sim L_{\text {device}}$. Their amplitude is greater for $100 \mathrm{~nm}<L_{\text {device }}<300 \mathrm{~nm}$ but drastically diminishes beyond that. This suggests that the mean free path is above $100 \mathrm{~nm}$ but clearly below $1 \mu \mathrm{m}$, in agreement with a rough estimate based on the carrier mobility [44] which yields $l \lesssim 100-260 \mathrm{~nm}$. Notice that the scaling of the period with $1 / L_{\text {device }}$ rules out Tomasch resonances [45-47] within YBCO, which should scale with its thickness and should therefore show the same period for all devices.

The short period oscillations, clearly visible for $V<\Delta$, follow $V_{\text {th,short }}=h v_{F} / 4 L_{\text {device. }}$. This period is expected from the interference of Andreev pairs [19,20]—scheme in Fig. 1(a). In this case the resonance condition reads $2 L_{\text {device }}\left|k_{1}-k_{2}\right|=2 n \pi$, where $k_{1}$ and $k_{2}$ are the electron and reflected hole wave vectors, whose difference is established by the applied voltage $\left|k_{1}-k_{2}\right|=2 V / \hbar v_{F}$ [20]. These resonances imply that the superconducting coherence is preserved over $\sim L_{\text {device}}$.

Our model also explains the gating effects. Figure 4(d) displays a simulated conductance as a function of bias and Fermi energy, which reproduces the main experimental features-particularly the pattern of oblique lines that characterize the conductance modulation by $V_{G}$ [Fig. 4(b)]. The correspondence between the $y$ axis in simulations and experiments implies that the Fermi energy (and vector $k_{F}$ ) is varied about proportionally to $V_{G}$, in agreement with earlier experiments [17]. Varying $k_{F}$ produces a periodic modulation of the conductance, via the resonance condition $2 L_{\text {device }} k_{F}=2 n \pi$, yielding the oblique lines. Note that the model also reproduces the slope gradual change, and the pronounced curvature over the plot's periphery, which is obtained by including in the model $\epsilon_{F, B}\left(V_{G}\right) \neq \epsilon_{F, A}\left(V_{G}\right)=$ $\epsilon_{F, C}\left(V_{G}\right)$ to account for a partial pining of graphene Fermi energy on conducting YBCO.

In summary, we have realized ballistic planar devices in which graphene is proximitized by a $d$-wave cuprate superconductor. We find that the $d$-wave correlations propagate into graphene over hundreds of $\mathrm{nm}$ at $T \sim 4 \mathrm{~K}$. The confinement of Andreev pairs and electrons in graphene homojunctions of submicrometric lateral dimensions produces quantum interferences that show as conductance oscillations. Those involving Andreev pairs, more prominent below the superconducting gap, are analogous to the de Gennes-Saint-James oscillations. Although difficult to observe in graphene-low-temperature superconductor devices, here they are very clear due to the large YBCO gap (tens of $\mathrm{mV}$ ), which fits many orders of interference within. The unusual $d$-wave Andreev-pair interferences are accompanied by normal-electron ones. The simultaneous observation of both is rare, and stems from the fact that the coherence length of $d$-wave correlations is limited by the mean free path [22]. Thus, the necessary condition for both types of resonances is satisfied simultaneously here.
Extensions of this study to graphene with lower intrinsic doping and higher carrier mobility should allow exploring specular Andreev reflection regimes [3] and Josephson effects. The latter would also require enhancing the transparency of the graphene-YBCO interface, possibly by replacing the $\mathrm{Au}$ interlayer, in order to routinely obtain low- $Z$ junctions. Further, perspectives include novel directional effects $[14,15]$ linked to the $d$-wave character of the induced correlations, which could be exploited in more sophisticated devices to realize topological states [48]. This work should also further encourage studies in which graphene is replaced by other 2D materials with intrinsically interesting proximity behavior $[49,50]$, eventually combined with exfoliated $d$-wave superconductors [51].

Work at Unité Mixte de Physique CNRS/Thales supported by the ERC Grant No. 647100 "SUSPINTRONICS," French ANR Grants No. ANR-15-CE24-0008-01 "SUPERTRONICS" and No. ANR-17-CE30-0018-04 "OPTOFLUXONICS," Labex NanoSaclay No. ANR-10LABX-0035, European COST action 16218 "Nanocohybri", European Union's H2020 Excellent Science (Grants No. 785219 and No. 881603), and JSPS core-to-core program A. Advanced Research Networks. This work was supported by the French RENATECH network (French national nanofabrication platform). X. P. Z., D. B., and F. S. B. acknowledge support from Ministerio de Ciencia e Innovacion (MICINN) through Project No. FIS2017-82804-P. F. S. B. acknowledges Grupos Consolidados UPV/EHU del Gobierno Vasco (Grant No. IT1249-19). We thank Professor J. Santamaria for discussions.

*javier.villegas@cnrs-thales.fr

[1] H. B. Heersche, P. Jarillo-Herrero, J. B. Oostinga, L. M. K. Vandersypen, and A. F. Morpurgo, Bipolar supercurrent in graphene, Nature (London) 446, 56 (2007).

[2] T. M. Klapwijk, Proximity effect from an Andreev perspective, J. Supercond. 17, 593 (2004).

[3] C. W. J. Beenakker, Specular Andreev Reflection in Graphene, Phys. Rev. Lett. 97, 067007 (2006).

[4] D. K. Efetov, L. Wang, C. Handschin, K. B. Efetov, J. Shuang, R. Cava, T. Taniguchi, K. Watanabe, J. Hone, C. R. Dean, and P. Kim, Specular interband Andreev reflections at van der Waals interfaces between graphene and $\mathrm{NbSe}_{2}$, Nat. Phys. 12, 328 (2015).

[5] M. T. Allen, O. Shtanko, I. C. Fulga, A. R. Akhmerov, K. Watanabe, T. Taniguchi, L. S. Levitov, and A. Yacoby, Spatially resolved edge currents and guided-wave electronic states in graphene, Nat. Phys. 12, 128 (2016).

[6] M. Ben Shalom, M. J. Zhu, V. I. Fal, A. Mishchenko, A. V. Kretinin, K. S. Novoselov, C. R. Woods, K. Watanabe, T. Taniguchi, A. K. Geim, and J. R. Prance, Proximity superconductivity in ballistic graphene, from Fabry-Perot oscillations to random Andreev states in magnetic field, Nat. Phys. 12, 318 (2016). 
[7] F. Amet, C. T. Ke, I. V. Borzenets, Y.-M. Wang, K. Watanabe, T. Taniguchi, R. S. Deacon, M. Yamamoto, Y. Bomze, S. Tarucha, and G. Finkelstein, Supercurrent in the quantum Hall regime, Science 352, 966 (2016).

[8] G. Koren, T. Kirzhner, Y. Kalcheim, and O. Millo, Signature of proximity-induced $p x+i p y$ triplet pairing in the doped topological insulator $\mathrm{Bi}_{2} \mathrm{Se}_{3}$ by the s-wave superconductor NbN, Europhys. Lett. 103, 67010 (2013).

[9] A. D. K. Finck, C. Kurter, Y. S. Hor, and D. J. Van Harlingen, Phase Coherence and Andreev Reflection in Topological Insulator Devices, Phys. Rev. X 4, 041022 (2014).

[10] A. D. K. Finck, C. Kurter, E. D. Huemiller, Y. S. Hor, and D. J. Van Harlingen, Robust Fabry-Perot interference in dual-gated $\mathrm{Bi}_{2} \mathrm{Se}_{3}$ devices, Appl. Phys. Lett. 108,203101 (2016).

[11] E. Bocquillon, R. S. Deacon, J. Wiedenmann, P. Leubner, T. M. Klapwijk, C. Brüne, K. Ishibashi, H. Buhmann, and L. W. Molenkamp, Gapless Andreev bound states in the quantum spin Hall insulator HgTe, Nat. Nanotechnol. 12, 137 (2017).

[12] J. Linder and A. Sudbø, Dirac Fermions and Conductance Oscillations in s- and $d$-Wave Superconductor-Graphene Junctions, Phys. Rev. Lett. 99, 147001 (2007).

[13] J. Linder and A. Sudbø, Tunneling conductance in $s$ - and $d$-wave superconductor-graphene junctions: Extended Blonder-Tinkham-Klapwijk formalism, Phys. Rev. B 77, 064507 (2008).

[14] J. Linder, A. M. Black-Schaffer, T. Yokoyama, S. Doniach, and A. Sudbø, Josephson current in graphene: Role of unconventional pairing symmetries, Phys. Rev. B 80, 094522 (2009).

[15] O. A. Awoga and A. M. Black-Schaffer, Probing unconventional superconductivity in proximitized graphene by impurity scattering, Phys. Rev. B 97, 214515 (2018).

[16] A. Di Bernardo, O. Millo, M. Barbone, H. Alpern, Y. Kalcheim, U. Sassi, A. K. Ott, D. De Fazio, D. Yoon, M. Amado, A. C. Ferrari, J. Linder, and J. W. A. Robinson, $P$-wave triggered superconductivity in single-layer graphene on an electron-doped oxide superconductor, Nat. Commun. 8, 14024 (2017).

[17] D. Perconte, F. A. Cuellar, C. Moreau-Luchaire, M. Piquemal-Banci, R. Galceran, P. R. Kidambi, M. B. Martin, S. Hofmann, R. Bernard, B. Dlubak, P. Seneor, and J. E. Villegas, Tunable Klein-like tunnelling of high-temperature superconducting pairs into graphene, Nat. Phys. 14, 25 (2018).

[18] A. F. Young and P. Kim, Quantum interference and Klein tunnelling in graphene heterojunctions, Nat. Phys. 5, 222 (2009).

[19] P. G. de Gennes and D. Saint-James, Elementary excitations in the vicinity of a normal metal-superconducting metal contact, Phys. Lett. 4, 151 (1963).

[20] J. M. Rowell and W. L. McMillan, Electron Interference in a Normal Metal Induced by Superconducting Contracts, Phys. Rev. Lett. 16, 453 (1966).

[21] T. Tsuneto, On dirty superconductors, Prog. Theor. Phys. 28, 857 (1962).

[22] A. V. Balatsky, I. Vekhter, and J. X. Zhu, Impurity-induced states in conventional and unconventional superconductors, Rev. Mod. Phys. 78, 373 (2006).
[23] W. Liang, M. Bockrath, D. Bozovic, J. H. Hafner, M. Tinkham, and H. Park, Fabry-Perot interference in a nanotube electron waveguide, Nature (London) 411, 665 (2001).

[24] F. Miao, S. Wijeratne, Y. Zhang, U. C. Coskun, W. Bao, and C. N. Lau, Phase-coherent transport in graphene quantum billiards, Science 317, 1530 (2007).

[25] L. C. Campos, A. F. Young, K. Surakitbovorn, K. Watanabe, T. Taniguchi, and P. Jarillo-Herrero, Quantum and classical confinement of resonant states in a trilayer graphene FabryPérot interferometer, Nat. Commun. 3, 1239 (2012).

[26] M. T. Allen, O. Shtanko, I. C. Fulga, J. I. J. Wang, D. Nurgaliev, K. Watanabe, T. Taniguchi, A. R. Akhmerov, P. Jarillo-Herrero, L. S. Levitov, and A. Yacoby, Observation of electron coherence and Fabry-Perot standing waves at a graphene edge, Nano Lett. 17, 7380 (2017).

[27] F. Giazotto, P. Pingue, F. Beltram, M. Lazzarino, D. Orani, S. Rubini, and A. Franciosi, Resonant Transport in $\mathrm{Nb} /$ GaAs/AlGaAs Heterostructures: Realization of the de Gennes-Saint-James Model, Phys. Rev. Lett. 87, 216808 (2001).

[28] P. R. Kidambi, C. Ducati, B. Dlubak, D. Gardiner, R. S. Weatherup, M. Martin, P. Seneor, H. Coles, and S. Hofmann, The parameter space of graphene CVD on polycrystalline Cu, J. Phys. Chem. C 116, 22492 (2012).

[29] B. Dlubak, P. R. Kidambi, R. S. Weatherup, S. Hofmann, and J. Robertson, Substrate-assisted nucleation of ultra-thin dielectric layers on graphene by atomic layer deposition, Appl. Phys. Lett. 100, 173113 (2012).

[30] S. Mzali, A. Montanaro, S. Xavier, B. Servet, J. P. Mazellier, O. Bezencenet, P. Legagneux, M. Piquemal-Banci, R. Galceran, B. Dlubak, P. Seneor, M. B. Martin, S. Hofmann, J. Robertson, C. S. Cojocaru, A. Centeno, and A. Zurutuza, Stabilizing a graphene platform toward discrete components, Appl. Phys. Lett. 109, 253110 (2016).

[31] G. E. Blonder, M. Tinkham, and T. M. Klapwijk, Transition from metallic to tunneling regimes in superconducting micro-constrictions: Excess current, charge imbalance, and super-current conversion, Phys. Rev. B 25, 4515 (1982).

[32] I. Maggio-Aprile, C. Renner, A. Erb, E. Walker, and Ø. Fischer, Direct Vortex Lattice Imaging and Tunneling Spectroscopy of Flux Lines on $\mathrm{YBa}_{2} \mathrm{Cu}_{3} \mathrm{O}_{7-\delta}$, Phys. Rev. Lett. 75, 2754 (1995).

[33] J. Y. T. Wei, N.-C. Yeh, D. F. Garrigus, and M. Strasik, Directional Tunneling and Andreev Reflection on $\mathrm{YBa}_{2} \mathrm{Cu}_{3} \mathrm{O}_{7-\delta}$ Single Crystals: Predominance of $d$-Wave Pairing Symmetry Verified with the Generalized Blonder, Tinkham, and Klapwijk Theory, Phys. Rev. Lett. 81, 2542 (1998).

[34] Y. Dagan, R. Krupke, and G. Deutscher, Determination of the superconducting gap in by tunneling experiments under magnetic fields, Phys. Rev. B 62, 146 (2000).

[35] V. Rouco, R. El Hage, A. Sander, J. Grandal, K. Seurre, X. Palermo, J. Briatico, S. Collin, J. Trastoy, K. Bouzehouane, A. I. Buzdin, G. Singh, N. Bergeal, C. Feuillet-Palma, J. Lesueur, C. Leon, M. Varela, J. Santamaría, and J. E. Villegas, Quasiparticle tunnel electroresistance in superconducting junctions, Nat. Commun. 11, 658 (2020).

[36] See Supplemental Material at http://link.aps.org/ supplemental/10.1103/PhysRevLett.125.087002 for details 
on the theoretical model, simulation procedure, fitting parameters, simulations vs. experiment for full set of devices, $d$-wave vs $s$-wave expectations, and supplemental references.

[37] S. Kashiwaya, Y. Tanaka, M. Koyanagi, H. Takashima, and $\mathrm{K}$. Kajimura, Origin of zero-bias conductance peaks in high$T_{c}$ superconductors, Phys. Rev. B 51, 1350 (1995).

[38] A. Sharoni, G. Koren, and O. Millo, Correlation of tunneling spectra with surface nanomorphology and doping in thin $\mathrm{YBa}_{2} \mathrm{Cu}_{3} \mathrm{O}_{7-\delta}$ films, Europhys. Lett. 54, 675 (2001).

[39] S. Kashiwaya, Y. Tanaka, M. Koyanagi, and K. Kajimura, Theory for tunneling spectroscopy of anisotropic superconductors, Phys. Rev. B 53, 2667 (1996).

[40] E. Scheer, W. Belzig, Y. Naveh, M. H. Devoret, D. Esteve, and C. Urbina, Proximity Effect and Multiple Andreev Reflections in Gold Atomic Contacts, Phys. Rev. Lett. 86, 284 (2001).

[41] A. Sharoni, I. Asulin, G. Koren, and O. Millo, Proximity Effect in Gold-Coated YBCO Films Studied by Scanning Tunneling Spectroscopy, Phys. Rev. Lett. 92, 017003 (2004).

[42] C. Hwang, D. A. Siegel, S.-K. Mo, W. Regan, A. Ismach, Y. Zhang, A. Zettl, and A. Lanzara, Fermi velocity engineering in graphene by substrate modification, Sci. Rep. 2, 590 (2012).

[43] A. H. Castro Neto, F. Guinea, N. M. R. Peres, K. S. Novoselov, and A. K. Geim, The electronic properties of graphene, Rev. Mod. Phys. 81, 109 (2009).
[44] E. H. Hwang and S. Das Sarma, Acoustic phonon scattering limited carrier mobility in two-dimensional extrinsic graphene, Phys. Rev. B 77, 115449 (2008).

[45] W. J. Tomasch, Geometrical Resonance and Boundary Effects in Tunneling from Superconducting In, Phys. Rev. Lett. 16, 16 (1966).

[46] O. Nesher and G. Koren, Observation of Tomasch oscillations and tunneling-like behavior in oxygen-deficient edge junctions, Appl. Phys. Lett. 74, 3392 (1999).

[47] C. Visani, Z. Sefrioui, J. Tornos, C. Leon, J. Briatico, M. Bibes, A. Barthélémy, J. Santamaría, and J. E. Villegas, Equal-spin Andreev reflection and long-range coherent transport in high-temperature superconductor/half-metallic ferromagnet junctions, Nat. Phys. 8, 539 (2012).

[48] P. Lucignano, F. Tafuri, and A. Tagliacozzo, Topological rf SQUID with a frustrating $\pi$ junction for probing the Majorana bound state, Phys. Rev. B 88, 184512 (2013).

[49] D. Kuzmanovski, J. Linder, and A. Black-Schaffer, Quantum ground state control in superconductor-silicene structures: $0-\pi$ transitions, $\varphi 0$-junctions, and Majorana bound states, Phys. Rev. B 94, 180505 (2016).

[50] J. Linder and T. Yokoyama, Anisotropic Andreev reflection and Josephson effect in ballistic phosphorene, Phys. Rev. B 95, 144515 (2017).

[51] A. J. Li, X. Zhu, G. R. Stewart, and A. F. Hebard, Bi-2212/1T-TaS 2 Van der Waals junctions: Interplay of proximity induced high- $T_{c}$ superconductivity and CDW order, Sci. Rep. 7, 1 (2017). 